\title{
Mudança de Eixos e Símbolos Projetivos (")
}

\author{
EDUARDO A. SALGADO
}

Escola Superior de Agricultura «Luiz de Queiroz»

(*) Recebido para publicação em $14 / 6 / 60$. 


\section{1 - INTRODUÇÃO}

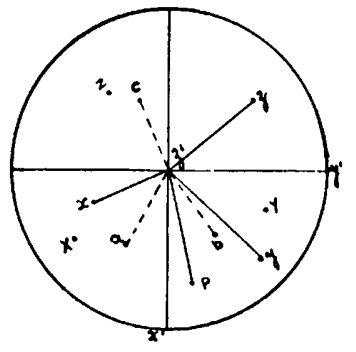

flo 1

Conhecido o símbolo mileriano de uma face, em um sistema de eixos cristalográficos, pode-se determinar o sín.. bolo da mesma face em outro sistema, mediante o uso de fórmulas adequadas à mudança de eixos coordenados e cuja dedução se encontra em tratados de cristalografia.

Vamos deduzir tais expressões, valendo-nos dos símbolos projetivos e dos cosenos de Wulff.

\section{2 - DEDUÇÃO}

$\mathrm{Na}$ figura 1 representam-se, em projeção estereográfica, os dois sistemas $X Y Z$ e $x y z$ e ainda o sistema retangular $x^{\prime} y^{\prime} z^{\prime}$, utilizado na determinação dos símbolos projetivos.

A face de pólo $P$ tem, no sistema $X Y Z$, o símbolo (qrs), no sistema $x y z$ o símbolo $\left(\mathrm{q}_{1} \mathrm{r}_{1} \mathrm{~s}_{1}\right)$ e o símbolo projetivo ( $\left.\mathrm{q}^{\prime} \mathrm{r}^{\prime} \mathrm{s}^{\prime}\right)$.

Os pontos $a, b, c$, representam, respectivamente, os pólos dos círculos máximos $Y Z, X Z, X Y$ e as coordenadas esféricas $\varphi$ são tomadas a partir de $y^{\prime}$.

Sejam $m, n, p$ os cosenos diretores da face parametral do sistema $x y z$, neste sistema.

Temos, pelo teorema dos cosenos de Wulff:

$\mathrm{q}_{1}=\frac{\cos \rho \mathrm{X} \cdot \cos \rho \mathrm{P}+\operatorname{sen} \rho \mathrm{X} \cdot \operatorname{sen} \rho \mathrm{P} \cdot \cos (\varphi \mathrm{X}-\varphi \mathrm{P})}{\mathrm{m}} \ldots$

$r_{1}=\frac{\cos \rho y \cos \rho P+\operatorname{sen} \rho y \cdot \operatorname{sen} \rho P \cdot \cos (\varphi P-\varphi y)}{n}$

$s_{1}=\frac{\cos \rho z \cdot \cos \rho P+\operatorname{sen} \rho z \cdot \operatorname{sen} \rho P \cdot \cos (\varphi P-\varphi z)}{P}$

$\mathrm{p}$

O plano $Y Z$, cujo símbolo é (100) em $X Y Z$, passa a ter os seguintes indices em $x y z$ :

$\mathrm{h}_{1}=\frac{\cos \rho \mathrm{X} \cdot \cos \rho \mathrm{a}+\operatorname{sen} \rho \mathrm{x} \cdot \operatorname{sen} \rho \mathrm{a} \cdot \cos (\varphi \mathrm{X}-\varphi \mathrm{a})}{\mathrm{m}} \ldots$
$\mathrm{k}_{1}=\frac{\cos \rho \mathrm{y} \cdot \cos \rho \mathrm{a}+\operatorname{sen} \rho \mathrm{y} \cdot \operatorname{sen} \rho \mathrm{a} \cdot \cos (\varphi \mathrm{a}-\varphi \mathrm{y})}{\mathrm{n}}$
$\mathrm{l}_{1}=\frac{\cos \rho \mathrm{z} \cdot \cos \rho \mathrm{a}+\operatorname{sen} \rho \mathrm{z} \cdot \operatorname{sen} \rho \mathrm{a} \cdot \cos (\varphi \mathrm{a}-\varphi \mathrm{z})}{\mathrm{p}}$ 
Os planos $X Z(010)$ e $X Y(001)$ darão expressões idênticas para $\left(h_{2} k_{2} l_{2}\right)$ e $\left(h_{3} k_{3} l_{3}\right)$.

De (1) tira-se:

$\mathrm{q}_{1}=\frac{\cos \rho \mathrm{X}}{\mathrm{m}} \cdot \cos \rho \mathrm{P}+\frac{\operatorname{sen} \rho \mathrm{X} \cdot \cos \varphi \mathrm{X}}{\mathrm{m}} \cdot \operatorname{sen} \rho \mathrm{P} \cdot \cos \varphi \mathrm{P}+$

$+\frac{\operatorname{sen} \rho X \cdot \operatorname{sen} \varphi X}{m} \cdot \operatorname{sen} \rho P \cdot \operatorname{sen} \varphi P$.

De (2) obtém-se, idênticamente:

$h_{1}=\frac{\cos \rho X}{m} \cdot \cos \rho a+\frac{\operatorname{sen} \rho X \cdot \cos \varphi X}{m} \cdot \operatorname{sen} \rho a \cdot \cos \varphi a+$ $+\frac{\operatorname{sen} \rho X \cdot \operatorname{sen} \varphi X}{m} \cdot \operatorname{sen} \rho \mathbf{a} \cdot \operatorname{sen} \varphi \mathrm{a}$ e expressões semelhantes para $h_{2}$ e $h_{3}$, podem ser conseguidas.

Façamos $\frac{\cos \rho X}{m}=A ; \frac{\operatorname{sen} \rho X \cdot \cos \varphi X}{m}=B ;$

$\frac{\operatorname{sen} \rho X \cdot \operatorname{sen} \varphi x}{m}=C$.

Podemos escrever, então:

$q_{1}=A \cdot \cos \rho P+B \cdot \operatorname{sen} \rho P \cdot \cos \varphi P+C \cdot \operatorname{sen} \rho P \cdot \operatorname{sen} \varphi P$

$h_{1}=A \cdot \cos \rho a+B \cdot \operatorname{sen} \rho \mathbf{a} \cdot \cos \varphi \mathrm{a}+\cdot C \cdot \operatorname{sen} \rho \mathbf{a} \cdot \operatorname{sen} \varphi \mathrm{a}$

$\mathrm{h}_{2}=\mathrm{A} \cdot \cos \rho \mathrm{b}+\mathrm{B} \cdot \operatorname{sen} \rho \mathrm{b} \cdot \cos \varphi \mathrm{b}+\mathrm{C} \cdot \operatorname{sen} \rho \mathrm{b} \cdot \operatorname{sen} \varphi \mathrm{b}$

$\mathrm{h}_{3}=\mathrm{A} \cdot \cos \rho \mathrm{C}+\mathrm{B} \cdot \operatorname{sen} \rho \mathrm{C} \cdot \cos \varphi \mathrm{C}+\mathrm{C} \cdot \operatorname{sen} \rho \mathrm{c} \cdot \operatorname{sen} \varphi \mathrm{C}$

Nestas expressões ponhamos em evidência $\operatorname{sen}_{\rho} \mathrm{P}$ e os valores que lhe correspondem: Temos:

$\left.\mathrm{q}_{1_{1}}=\operatorname{sen} \rho \mathrm{P} \mid \mathrm{A} \cdot \operatorname{cotg} \rho \mathrm{P}+\mathrm{B} \cdot \cos \varphi \mathrm{P}+\mathrm{C} \cdot \operatorname{sen} \varphi \mathrm{P}\right] \ldots$ (3)

$\mathrm{h}_{1}=\operatorname{sen} \rho \mathrm{a} \quad[\mathrm{A} \cdot \operatorname{cotg} \rho \mathrm{a}+\mathrm{B} \cdot \cos \varphi \mathrm{a}+\mathrm{C} \cdot \operatorname{sen} \varphi \mathrm{a}] \ldots$ (4)

$\mathrm{h}_{2}=\operatorname{sen} \rho \mathrm{b} \quad[\mathrm{A} \cdot \operatorname{cotg} \rho \mathrm{b}+\mathrm{B} \cdot \cos \varphi \mathrm{b}+\mathrm{C} \cdot \operatorname{sen} \varphi \mathrm{b}] \ldots(5)$

$\mathrm{h}_{3}=\operatorname{sen} \rho \mathrm{C} \quad[\mathrm{A} \cdot \operatorname{cotg} \rho \mathrm{C}+\mathrm{B} \cdot \cos \varphi \mathrm{C}+\mathrm{C} \cdot \operatorname{sen} \varphi \mathrm{C}] \ldots(6)$

Em virtude do teorema 3 dos simbolos projetivos (BOLDY. REV, (1934) a expressão (3) transforma-se em:

$$
\mathrm{q}_{1}=\operatorname{sen} \rho \mathrm{P}\left\{\mathrm{As}^{\prime}+\mathrm{Br}^{\prime}+\mathrm{Cq}^{\prime}\right\} \text {. }
$$

De acôrdo com a expressão [13]da página 151 da Cristalografia atrás mencionada, os planos (100), (010) e (001) em XYZ terão para símbolos projetivos, respectivamente, $\mathrm{s}_{1} \mathrm{~s}_{2} \mathrm{~s}_{3} ; \mathrm{t}_{1} \mathrm{t}_{2} \mathrm{t}_{3} ; \mathrm{u}_{1} \mathrm{u}_{2} \mathrm{u}_{3}$ e as expressões (4), (5) e (6) mudam para: 


$$
\begin{aligned}
& \mathrm{h}_{1}=\operatorname{sen} \rho \mathrm{a}\left\{\mathrm{As}_{3}+\mathrm{Bs}_{2}+\mathrm{Cs}_{1}\right\} \ldots \ldots \ldots \ldots \ldots \\
& \mathrm{h}_{2}=\operatorname{sen} \rho \mathrm{b}\left\{\mathrm{At}_{3}+\mathrm{Bt}_{2}+\mathrm{Ct}_{1}\right\} \ldots \ldots \ldots \ldots \ldots
\end{aligned}
$$

$\mathrm{Na}$ expressão (7) substituam-se os indices projetivos pelos seus respectivos valores, obtidos da expressão [13] de Boldyrev. Obtém-se:

$$
\begin{gathered}
q_{1}=\operatorname{sen} \rho P\left\{\begin{array}{c}
A\left(\mathrm{qs}_{3}+\mathrm{rt}_{3}+\mathrm{su}_{3}\right)+\mathrm{B}\left(\mathrm{qs}_{2}+\mathrm{rt}_{2}+\right. \\
\left.+\mathrm{su}_{2}\right)+\mathrm{C}\left(\mathrm{qs}_{1}+\mathrm{rt}_{1}+\mathrm{su}_{1}\right)
\end{array}\right\}= \\
=\operatorname{sen} \rho \mathrm{P}\left\{\begin{array}{c}
\mathrm{q}\left(\mathrm{As}_{3}+\mathrm{Bs}_{2}+\mathrm{Cs}_{1}\right)+\mathrm{r}\left(\mathrm{At}_{3}+\mathrm{Bt}_{2} \times \mathrm{Ct}_{1}\right)+ \\
+\mathrm{s}\left(\mathrm{Au}_{3}+\mathrm{Bu}_{2}+\mathrm{Cu}_{1}\right) \ldots \ldots \ldots \ldots(11)
\end{array}\right] \\
\text { De (11) tira-se, levando em conta (8), (9) e (10): }
\end{gathered}
$$

$$
q_{1}=\operatorname{sen} \rho P\left\{\frac{h_{1} q}{\operatorname{sen} \rho a}+\frac{h_{2} r}{\operatorname{sen} \rho b}+\frac{h_{s} s}{\operatorname{sen} \rho c}\right\} ，
$$

obtendo-se expressões idênticas para $r_{1}$ e $s_{1}$.

Dividindo por $\operatorname{sen} \rho P$ e fazendo $\frac{1}{\operatorname{sen} \rho a}=L, \frac{1}{\operatorname{sen} \rho b}=L$, $\frac{1}{\operatorname{sen} \rho c}=L "$ chega-se às expressões obtidas por BUTTGENBACH, (1935):

$$
\begin{aligned}
& \mathrm{q}_{1}=\mathrm{Lh}_{1} \mathrm{q}+\mathrm{L}^{\prime} \mathrm{h}_{2} \mathrm{r}+\mathrm{L}^{\prime \prime} \mathrm{h}_{3} \mathrm{~S} \\
& r_{1}=L k_{1} q+L^{\prime} k_{2} r+L^{\prime \prime} k_{3} S \\
& \mathrm{~s}_{1}=\mathrm{Ll}_{1} \mathrm{q}+\mathrm{L}^{\prime} \mathrm{l}_{2} \mathrm{r}+\mathrm{L}^{\prime \prime} \mathrm{l}_{\mathrm{s}} \mathrm{S} \\
& \text { 3. - RESUMO }
\end{aligned}
$$

O presente trabalho apresenta uma nova dedução das expressões que servem, em cristalografia, à mudança de eixos coordenados, valendo-se o autor da projeção estereográfica. 


\section{4. - SUMMARY}

The present work presents a new deduction of the expressions which are used to the change of the coordinate axes in Crystallography, the author using the stereographic projection.

\section{5 - LITERATURA CITADA}

BOLDYREV, A.K.-1934 - Cristalografia - tradução do russo para o espanhol por Rafael Candel Vila - Editorial Labor.

BUTTGENBACH, H. H.-1935 - Les minéraux et les roches - Dunod, Paris, p. 321. 
